\title{
The Role of Salvage High-Dose Chemotherapy in Relapsed Male Germ Cell Tumors
}

\author{
Christoph Oing ${ }^{a}$ Anja Lorch ${ }^{b}$ \\ ${ }^{a}$ Department of Oncology, Hematology and Bone Marrow Transplantation with Section of Pneumology, University Medical Center \\ Hamburg-Eppendorf, Hamburg, Germany;

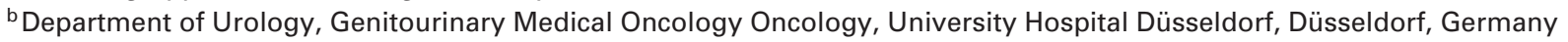

\section{Keywords}

Germ cell tumor - Salvage chemotherapy .

High-dose chemotherapy · Carboplatin · Etoposide ·

Treatment-related mortality

\section{Summary}

Germ cell tumors (GCT) are a unique tumor entity with excellent cure rates if guideline-endorsed treatment is thoroughly applied. Even patients with widespread metastatic disease can often be cured with cisplatin-based combination chemotherapy as part of a multimodal treatment approach. However, about $30 \%$ of patients with metastatic disease at initial presentation, corresponding to about $5-10 \%$ of all GCT patients, relapse or progress despite first-line treatment and therefore require salvage chemotherapy. Salvage systemic treatment either consists of conventional-dose cisplatin-based combination chemotherapy or sequential high-dose treatment with carboplatin and etoposide plus subsequent autologous stem cell support. This review is based on a comprehensive literature search of MEDLINE and conference proceedings of ESMO, ASCO, and EAU meetings until 2018 and provides an overview of current treatment options for germ cell cancer patients relapsing after or progressing during firstline cisplatin-based combination chemotherapy.

(C) 2018 S. Karger GmbH, Freiburg

\section{Introduction}

Germ cell tumors (GCT) are the most common solid malignancy in men aged $\leq 45$ years [1]. Since the successful implementation of cisplatin-based combination chemotherapy, $50-90 \%$ of patients with advanced disease (based on the presence or absence of clinical risk factors according to the IGCCCG (International Germ Cell Cancer Collaborative Group score) can be cured successfully, and GCT have become a unique model of a curable malignancy [2-4]. Nonetheless, about $30 \%$ of patients with metastatic disease at initial presentation, corresponding to about $5-10 \%$ of all GCT patients, relapse despite proper guideline-endorsed first-line multimodal treatment, with the need for further therapy. First salvage treatment generally consists of either conventional-dose or high-dose chemotherapy (HD-CT) plus subsequent autologous stem cell support. With multimodal salvage treatment including HD-CT, about $50 \%$ of salvage patients still have a chance to achieve complete remission and disease-free long-term survival, compared to only about $25 \%$ of patients treated with conventional-dose chemotherapy $[5,6]$. HD-CT represents dose-intensified cytostatic treatment with accompanying enhanced toxicity, mainly of hematological origin, which is counteracted by autologous stem cell support that significantly reduces the time to hematopoietic recovery [7]. However, to date, the use of HD-CT as first salvage is still a matter of controversy because of partly unequivocal study results [8].

\section{Methods}

This review will provide a comprehensive and concise summary of current indications for salvage HD-CT approaches in patients responding inadequately to first-line chemotherapy or relapsing after platinum-based conventional-dose first salvage chemotherapy.

A selective literature search of MEDLINE was conducted to identify clinical trials and large retrospective analyses reporting on first and subsequent salvage HD-CT approaches for the treatment of relapsed GCT published from 1990 until the present. Conference proceedings of ASCO, EAU, and ESMO annual meetings from 2015 to 2018 were searched for unpublished data on clinical

\section{KARGER}

() 2018 S. Karger GmbH, Freiburg

Fax +497614520714 
trials and retrospective analyses. Furthermore, review papers were hand-searched to identify other meaningful references. In order to provide high-evidence literature, case reports and small singlecenter retrospective series were excluded.

\section{Results}

In general, the interpretability of trial results and the level of evidence with regard to salvage HD-CT as treatment for relapsed and/or refractory GCT are limited, since patient numbers were commonly small, study populations were often highly heterogeneous with respect to GCT subtypes and/or previous treatments, and high-evidence data from randomized trials are still lacking.

\section{Prognostic Factors for First Salvage Chemotherapy - the IPFSG Score}

Primary treatment decisions for advanced GCT are mainly based on the IGCCCG risk stratification model [2]. Similar clinical predictive factors could also be identified for GCT patients failing routine first-line treatment. Based on a large international retrospective database analysis of approximately 1,600 patients by the International Prognostic Factors Study Group (IPFSG), 5 different risk groups (very low, low, intermediate, high, very high) stratified by 7 different clinical characteristics could be identified with a predicted long-term progression-free survival (PFS) ranging from less than $10 \%$ to approximately $75 \%$ [9]. The independent prognostic variables identified were: primary histology, primary tumor location, response to first-line treatment, progression-free interval following first-line treatment, serum tumor marker elevation (alphafetoprotein and/or human chorionic gonadotropin), and non-pulmonary visceral metastases at relapse. The IPFSG score risk assessment may not only help to predict individual prognosis but also aid treatment decision making at first relapse.

\section{First Salvage Conventional-Dose Chemotherapy}

Long-term remissions following conventional salvage chemotherapy have been reported in about $15-60 \%$ of patients. Ifosfamide and cisplatin comprise the backbone of the most effective conventional salvage regimens, combined with etoposide (VIP) [10], vinblastine (VeIP) [6], or paclitaxel (TIP) [11, 12], with none of these regimens being clearly superior and direct randomized comparisons lacking. All combinations are generally applied for 4 cycles repeated every 21 days. Table 1 provides an overview of common first salvage conventional regimens.

\section{First Salvage HD-CT}

The beneficial effect of HD-CT as first salvage strategy is still a matter of debate. A retrospective matched pair analysis published in 2002 demonstrated a $10 \%$ advantage with respect to both eventfree survival (EFS) and overall survival (OS) in favor of early highdose salvage treatment [13].

Subsequent studies confirmed the beneficial impact. A retrospective analysis of 135 patients treated at Indiana University dis-
Table 1. Conventional salvage chemotherapy regimens

\begin{tabular}{llll}
\hline Regimen/agent & Dose & Days & Reference \\
\hline VIP & & & \\
Etoposide & $75 \mathrm{mg} / \mathrm{m}^{2}$ & $1-5$ & {$[10]$} \\
Ifosfamide & $1,200 \mathrm{mg} / \mathrm{m}^{2}$ & $1-5$ & \\
$\quad$ Cisplatin & $20 \mathrm{mg} / \mathrm{m}^{2}$ & $1-5$ & \\
VeIP & & & \\
Vinblastin & $0.11 \mathrm{mg} / \mathrm{kg}^{2}$ & $1+2$ & {$[6]$} \\
Ifosfamide & $1,200 \mathrm{mg} / \mathrm{m}^{2}$ & $1-5$ & \\
Cisplatin & $20 \mathrm{mg} / \mathrm{m}^{2}$ & $1-5$ & \\
TIP & & & \\
Paclitaxel & $175-200 \mathrm{mg} / \mathrm{m}^{2}$ & 1 & \\
Ifosfamide & $1,200 \mathrm{mg} / \mathrm{m}^{2}$ & $1-5$ & \\
Cisplatin & $20 \mathrm{mg} / \mathrm{m}^{2}$ & $1-5$ & \\
\hline
\end{tabular}

played a 70\% survival benefit in patients undergoing HD-CT across all risk categories [7]. A prospective trial of 81 relapsed patients with unfavorable prognostic markers reported a 5-year OS of 50\% following HD-CT [14]. Furthermore, a subgroup analysis of the IPFSG study cohort revealed a significant improvement in PFS and OS for all 5 prognostic subgroups (except for OS in the lowrisk group, most likely due to the low patient number) by about $10-15 \%$ in favor of HD-CT compared to conventional salvage treatment, and an approximate $10 \%$ OS benefit for the entire study population [15]. Of all 1,594 patients failing cisplatin-based firstline treatment, 773 and 821 patients underwent conventional- and high-dose salvage treatment, respectively. Subgroups were well balanced with a slightly higher number of patients with a favorable response to first-line therapy in the conventional treatment group, and slightly more patients with non-pulmonary visceral metastases in the high-dose treatment group. Several additional retrospective series including one with patients having bone metastases and a retrospective analysis of the German Testicular Cancer Study Group reporting on 143 patients undergoing either conventionaldose $(n=48)$ or high-dose $(n=95)$ therapy as first salvage could also confirm the superiority of HD-CT over conventional-dose therapy $[16,17]$. Recently, the group from Indiana University published long-term disease-free survival rates with HD-CT of 25, 33, and $40 \%$ of patients with either mediastinal primary, absolute platinum-refractory disease, or progressive brain metastases as poorrisk features [18]. Moreover, patients with unresectable late relapse after first- or further-line treatment showed very dismal outcomes with only $15 \%$ of patients achieving long-term PFS even after salvage $\mathrm{HD}-\mathrm{CT}[19]$.

In contrast to the aforementioned results from non-randomized studies, the randomized phase III trial 'IT-94' that prospectively compared conventional-dose chemotherapy to HD-CT failed to demonstrate a statistically significant difference between the 2 strategies. 280 relapsed GCT patients were randomized to receive either 4 cycles of VIP or VeIP or 3 such cycles followed by a single cycle of high-dose carboplatin, etoposide, and cyclophosphamide (HD-CEC). The 3-year EFS was better with HD-CEC (35 vs. 42\%) without reaching statistical significance, whereas OS was comparable in both arms [20]. It has to be taken into account that i) patient 
numbers were rather small, ii) many of the patients were lacking poor prognostic features, and iii) the high-dose approach does not reflect the current standard of care. Moreover, about one third of patients assigned to the HD-CEC arm did not receive the planned treatment for various reasons.

The question of conventional-dose chemotherapy versus $\mathrm{HD}-\mathrm{CT}$ is currently being addressed in a prospective randomized trial comparing 4 cycles of TIP versus sequential HD-CT (TIGER trial, NCT02375204) in a transatlantic multicenter collaborative effort between Alliance in the United States and EORTC in Europe.

\section{Defining the Optimal Salvage High-Dose Regimen}

Since the late 1980s, HD-CT has become an established option for first or subsequent salvage treatment. Since then, carboplatin and etoposide (HD-CE) have comprised the backbone of this treatment due to their effectiveness against GCT and predominantly hematological toxicity with otherwise non-hematological side effects (i.e., nephro-, oto-, and neurotoxicity) comparable to conventional-dose chemotherapy with cisplatin. The combination of HD-CE plus autologous stem cell support as well as improved supportive care has helped to reduce treatment-related mortality from about $10 \%$ to approximately $3 \%$ or less in the most recent series.

Further intensification of treatment to enhance cytotoxicity by adding a third agent, e.g., ifosfamide, cyclophosphamide, paclitaxel, gemcitabine, or thiotepa, did not improve outcomes meaningfully but resulted in a significant increase in adverse effects [21-25].

A comparison of single HD-CT using 3 drugs versus sequential HD-CT using the standard carboplatin and etoposide has been carried out in a large prospective, randomized, multicenter phase III trial by the German Testicular Cancer Study Group. A total of 216 patients with relapsed and/or refractory GCT were randomized to receive either 1 cycle of conventional VIP followed by 3 cycles of HD-CE (arm A) versus 3 cycles of VIP followed by a single cycle of high-dose carboplatin $2,200 \mathrm{mg} / \mathrm{m}^{2}$, etoposide $1,800 \mathrm{mg} / \mathrm{qm}^{2}$, and cyclophosphamide $6,400 \mathrm{mg} / \mathrm{m}^{2}$ (arm B). The study was stopped prematurely due to excess treatment-related mortality in the triplecombination HD-CEC arm. 1-year EFS, PFS, and OS rates did not differ significantly in both treatment arms with 40,53 , and $80 \%$ (arm A) versus 37, 49, and 61\% (arm B). Treatment-related deaths due to sepsis or cardiac toxicity were reported in $4 \%$ (arm A) versus $14 \%(\operatorname{arm~B})(\mathrm{p}<0.01)$ [26]. Long-term results of the same trial after 5 -years were as follows: PFS 47 versus $45 \%$; OS 49 versus $39 \%$ with a borderline significance for OS ( $\mathrm{p}=0.057$ ), which was mainly due to the higher number of early treatment-related deaths in the HD-CEC arm [27]. As a consequence, 2 or 3 cycles of HD-CE followed by autologous stem cell transplantation is the worldwide most commonly applied high-dose salvage regimen to date and can be used equivalently, according to current knowledge.

A recently published prospective phase II trial reported on 43 patients with relapsed or refractory disease that received an unusual tandem HD-CT approach of gemcitabine, docetaxel, melphalan, and carboplatin (GemDMC, first cycle) and ifosfamide, carboplatin, and etoposide (ICE, second cycle) with or without the anti-
Table 2. Established high-dose chemotherapy regimens

\begin{tabular}{llll}
\hline Regimen/agent & Dose & Days & Reference \\
\hline $\begin{array}{l}\text { Triple sequential approach } \\
\text { Carboplatin }\end{array}$ & AUC $8 \mathrm{mg} / \mathrm{ml} / \mathrm{min}$ & $1-3$ & [37] \\
$\begin{array}{l}\text { Etoposide } \\
\text { Double sequential approach }\end{array}$ & INDIANA & $1-3$ & \\
Carboplatin & $700 \mathrm{mg} / \mathrm{m}^{2}$ & & \\
Etoposide & $750 \mathrm{mg} / \mathrm{m}^{2}$ & $1-3$ & [7] \\
\hline
\end{tabular}

${ }^{a}$ Repeated every 21-28 days depending on hematologic recovery.

${ }^{b}$ Repeated at haematological recovery.

AUC $=$ Area under the curve.

vascular endothelial growth factor antibody bevacizumab. The objective response rate was high at $89 \%$ in an overall heterogeneous patient population (including $14 \%$ cisplatin-sensitive patients). As excess toxicity with a mortality rate of $9 \%$ was reported and patients receiving bevacizumab showed worse outcomes, bevacizumab-based HD-CT cannot be recommended [28].

Established sequential HD-CT regimens are displayed in table 2.

\section{Second or Subsequent HD-CT}

High-level evidence supporting the use of HD-CT as second- or further-line treatment is lacking. A retrospective study reported a favorable response rate of $55 \%$ after $\mathrm{HD}-\mathrm{CT}$ as second salvage treatment, and a remarkably long-term survival rate of $17 \%$ [29]. Moreover, a retrospective study by Einhorn et al. [7] found that only $45 \%$ of patients who received HD-CT with carboplatin and etoposide as third-line treatment stayed disease-free during followup as compared to $69 \%$ of patients who had HD-CE as first salvage treatment. Hence, HD-CT at second or further relapse seems to be inferior to early high-dose treatment. However, unless contraindicated, it is recommended even in patients who have failed to respond to multiple previous conventional treatments.

Unfortunately, randomized trials in this setting are lacking and will most likely never be conducted. Patients should be referred and critically evaluated at an expert center.

Role of Residual Tumor Resection Following Salvage HD-CT

Irrespective of the type and line of salvage systemic treatment, complete resection of all detectable residual masses is paramount for treatment success in terms of long-term survival. In retrospective series, the reported proportion of patients with viable cancer in resected masses who suffered a relapse and underwent salvage chemotherapy was as high as $70 \%$ [30-34].

\section{Discussion}

Cisplatin-resistant or -refractory disease is still one of the major challenges in GCT care. The prognosis of patients failing more than 2 lines of systemic treatment for advanced disease is very poor, and life expectancy with palliative chemotherapy is still limited to only a few months in the majority of patients [35]. 
With the use of salvage HD-CT followed by reinfusion of autologous hematopoietic stem cells, survival outcomes have been improved irrespective of clinical risk factors, and first-salvage HD-CE is therefore a valuable curative treatment approach. In further lines of treatment, the curative potential of even HD-CT is much lower. However, aggressive treatment including HD-CT should be thoroughly evaluated to provide the best possible care.

The available evidence is currently still limited, mainly because many studies i) were of retrospective design, ii) included only limited heterogeneous patient populations often at single centers, (iii) included patients with inadequate first-line treatment, or (iv) included patients who underwent consolidation HD-CT.

To substantially further improve the outcomes of cisplatin-resistant GCT patients, it is therefore imperative to provide adequate multimodal first-line treatment to prevent the development of chemoresistant disease, and to early identify patients with absolute cisplatin-refractory disease in order to intensify treatment in a timely manner. To this end, the referral of patients with advanced disease (preferably at first diagnosis) to centers with profound GCT expertise and inclusion in clinical trials whenever possible are paramount.

\section{Conclusion}

For now, the decision for or against a high-dose salvage approach should be thoroughly evaluated in all patients with relapsed and refractory GCT. Referral of these patients to expert centers is therefore strongly recommended. Outside a clinical trial, patients may be treated with conventional-dose chemotherapy or HD-CT depending on the presence or absence of adverse prognostic factors, availability of resources, and patient as well as physician preferences. The international prospective phase III trial TIGER (NCT02375204) will provide high evidence to finally answer the question of whether or not, and in which patients, HD-CT as first salvage treatment is superior to conventional treatment [36]. Hence, all patients should be included in this trial as soon as it opens for recruitment at German centers. To further improve the prognosis of relapsed or refractory disease, more preclinical and clinical research is urgently needed to overcome resistance mechanisms, enhance the effectiveness of systemic treatment, and lower treatment burden and treatment-associated morbidity and mortality.

\section{Disclosure Statement}

The authors declare that there is no conflict of interest to bias the development of the current manuscript.

\section{References}

1 Ruf CG, Isbarn H, Wagner W, et al.: Changes in epidemiologic features of testicular germ cell cancer: age at diagnosis and relative frequency of seminoma are constantly and significantly increasing. Urol Oncol 2014; 32:33 e31-36.

2 No authors listed: International Germ Cell Consensus Classification: a prognostic factor-based staging system for metastatic germ cell cancers. International Germ Cell Cancer Collaborative Group. J Clin Oncol 1997; 15:594-603.

3 Van Dijk MR, Steyerberg EW, Habbema JD: Survival of non-seminomatous germ cell cancer patients according to the IGCC classification: an update based on meta-analysis. Eur J Cancer 2006;42:820-826.

4 Horwich A, Shipley J, Huddart R: Testicular germ-cell cancer. Lancet 2006;367:754-765.

5 Feldman DR, Powles T: Salvage high-dose chemotherapy for germ cell tumors. Urol Oncol 2015;33:355-362.

6 Loehrer PJ Sr, Gonin R, Nichols CR, et al.: Vinblastine plus ifosfamide plus cisplatin as initial salvage therapy in recurrent germ cell tumor. J Clin Oncol 1998;16: 2500-2504.

7 Einhorn LH, Williams SD, Chamness A, et al.: Highdose chemotherapy and stem-cell rescue for metastatic germ-cell tumors. N Engl J Med 2007;357:340-348.

8 Beyer J, Albers P, Altena R, et al.: Maintaining success, reducing treatment burden, focusing on survivorship: highlights from the third European consensus conference on diagnosis and treatment of germ-cell cancer. Ann Oncol 2013;24:878-888.

9 International Prognostic Factors Study Group, Lorch A, Beyer J, et al.: Prognostic factors in patients with metastatic germ cell tumors who experienced treatment failure with cisplatin-based first-line chemotherapy. J Clin Oncol 2010;28:4906-4911.
10 Motzer RJ, Cooper K, Geller NL, et al.: The role of ifosfamide plus cisplatin-based chemotherapy as salvage therapy for patients with refractory germ cell tumors. Cancer 1990;66:2476-2481.

11 Motzer RJ, Sheinfeld J, Mazumdar M, et al.: Paclitaxel, ifosfamide, and cisplatin second-line therapy for patients with relapsed testicular germ cell cancer. J Clin Oncol 2000;18:2413-2418.

12 Kondagunta GV, Bacik J, Donadio A, et al.: Combination of paclitaxel, ifosfamide, and cisplatin is an effective second-line therapy for patients with relapsed testicular germ cell tumors. J Clin Oncol 2005;23:6549-6555.

13 Beyer J, Stenning S, Gerl A, et al.: High-dose versus conventional-dose chemotherapy as first-salvage treatment in patients with non-seminomatous germ-cell tumors: a matched-pair analysis. Ann Oncol 2002;13: 599-605.

14 Kondagunta GV, Bacik J, Sheinfeld J, et al.: Paclitaxel plus ifosfamide followed by high-dose carboplatin plus etoposide in previously treated germ cell tumors. J Clin Oncol 2007;25:85-90.

15 Lorch A, Bascoul-Mollevi C, Kramar A, et al.: Conventional-dose versus high-dose chemotherapy as first salvage treatment in male patients with metastatic germ cell tumors: evidence from a large international database. J Clin Oncol 2011;29:2178-2184.

16 Oing C, Lorch A, Bokemeyer C, et al.: First salvage treatment of germ cell tumor patients with bone metastases: retrospective analysis of a large international database. J Cancer Res Clin Oncol 2015;141:923-931.

17 Berger LA, Bokemeyer C, Lorch A, et al.: First salvage treatment in patients with advanced germ cell cancer after cisplatin-based chemotherapy: analysis of a registry of the German Testicular Cancer Study Group (GTCSG). J Cancer Res Clin Oncol 2014;140:1211-1220.
18 Adra N, Abonour R, Althouse SK, et al.: High-dose chemotherapy and autologous peripheral-blood stemcell transplantation for relapsed metastatic germ cell tumors: the Indiana University experience. J Clin Oncol 2017;35:1096-1102.

19 Lorch A, Rick O, Wundisch T, et al.: High dose chemotherapy as salvage treatment for unresectable late relapse germ cell tumors. J Urol 2010;184:168-173.

20 Pico JL, Rosti G, Kramar A, et al.: A randomised trial of high-dose chemotherapy in the salvage treatment of patients failing first-line platinum chemotherapy for advanced germ cell tumours. Ann Oncol 2005;16: 1152-1159.

21 Broun ER, Nichols CR, Tricot G, et al.: High dose carboplatin/VP-16 plus ifosfamide with autologous bone marrow support in the treatment of refractory germ cell tumors. Bone Marrow Transplant 1991;7:53-56.

22 Lotz JP, Bui B, Gomez F, et al.: Sequential high-dose chemotherapy protocol for relapsed poor prognosis germ cell tumors combining two mobilization and cytoreductive treatments followed by three high-dose chemotherapy regimens supported by autologous stem cell transplantation. Results of the phase II multicentric TAXIF trial. Ann Oncol 2005;16:411-418.

23 Margolin KA, Doroshow JH, Frankel P, et al.: Paclitaxel-based high-dose chemotherapy with autologous stem cell rescue for relapsed germ cell cancer. Biol Blood Marrow Transplant 2005;11:903-911.

24 Motzer RJ, Mazumdar M, Bosl GJ, et al.: High-dose carboplatin, etoposide, and cyclophosphamide for patients with refractory germ cell tumors: treatment results and prognostic factors for survival and toxicity. J Clin Oncol 1996;14:1098-1105. 
25 Selle F, Wittnebel S, Biron P, et al.: A phase II trial of high-dose chemotherapy (HDCT) supported by hematopoietic stem-cell transplantation (HSCT) in germ-cell tumors (GCTs) patients failing cisplatinbased chemotherapy: the Multicentric TAXIF II study. Ann Oncol 2014;25:1775-1782.

26 Lorch A, Kollmannsberger C, Hartmann JT, et al.; German Testicular Cancer Study Group: Single versus sequential high-dose chemotherapy in patients with relapsed or refractory germ cell tumors: a prospective randomized multicenter trial of the German Testicular Cancer Study Group. J Clin Oncol 2007;25:2778-2784.

27 Lorch A, Kleinhans A, Kramar A, et al.: Sequential versus single high-dose chemotherapy in patients with relapsed or refractory germ cell tumors: long-term results of a prospective randomized trial. J Clin Oncol 2012;30:800-805.
28 Nieto Y, Tu SM, Bassett R, et al.: Bevacizumab/highdose chemotherapy with autologous stem-cell transplant for poor-risk relapsed or refractory germ-cell tumors. Ann Oncol 2015;26:2507-2508.

29 Lorch A, Neubauer A, Hackenthal M, et al.: High-dose chemotherapy (HDCT) as second-salvage treatment in patients with multiple relapsed or refractory germ-cell tumors. Ann Oncol 2010;21:820-825.

30 Stephenson AJ, Bosl GJ, Motzer RJ, et al.: Retroperitoneal lymph node dissection for nonseminomatous germ cell testicular cancer: impact of patient selection factors on outcome. J Clin Oncol 2005;23:2781-2788.

31 Schirren J, Trainer S, Eberlein M, et al.: The role of residual tumor resection in the management of nonseminomatous germ cell cancer of testicular origin. Thorac Cardiovasc Surg 2012;60:405-412.

32 Heidenreich A, Ohlmann C, Hegele A, Beyer J: Repeat retroperitoneal lymphadenectomy in advanced testicular cancer. Eur Urol 2005;47:64-71.
33 McKiernan JM, Motzer RJ, Bajorin DF, et al.: Reoperative retroperitoneal surgery for nonseminomatous germ cell tumor: clinical presentation, patterns of recurrence, and outcome. Urology 2003;62:732-736.

34 Rick O, Bokemeyer C, Weinknecht S, et al.: Residual tumor resection after high-dose chemotherapy in patients with relapsed or refractory germ cell cancer. J Clin Oncol 2004;22:3713-3719.

35 Einhorn LH: Treatment of testicular cancer: a new and improved model. J Clin Oncol 1990;8:1777-1781.

36 Feldman DR, Huddart R, Hall E, et al.: Is high dose therapy superior to conventional dose therapy as initial treatment for relapsed germ cell tumors? The TIGER trial. J Cancer 2011;2:374-377.

37 Feldman DR, Sheinfeld J, Bajorin DF, et al.: TI-CE high-dose chemotherapy for patients with previously treated germ cell tumors: results and prognostic factor analysis. J Clin Oncol 2010;28:1706-1713. 